\title{
Calcium and vitamin D supplements administration in patients with nephrolithiasis; an observational prospective study
}

\author{
Marwan Ghazi Akel ${ }^{1,2^{*}}$, Iqbal Mohamad Fahs ${ }^{1 s}$ \\ Abstract \\ Introduction: Calcium and vitamin D supplements intake have been linked to nephrolithiasis as around $80 \%$ of kidney stones \\ contain calcium. However, studies regarding these supplements administration yielded conflicting data. \\ Objectives: In this study we aimed to assess calcium and vitamin D supplements intake and the presence of significant association \\ with nephrolithiasis among Lebanese patients. \\ Patients and Methods: The study was conducted at nephrology clinics selected randomly from all Lebanese districts over a 3-month \\ period. Data analysis was conducted with SPSS version 22. \\ Results: The mean age of the 550 studied participants was $42.12 \pm 15.59$ years with $51.5 \%$ males and $48.5 \%$ females. Almost half of \\ the participants had a history of kidney stones (53.8\%). Calcium and vitamin D supplements were utilized by $19.6 \%$ and $18.2 \%$, \\ respectively. Calcium and vitamin D supplements intake were not associated significantly with history of kidney stones (calcium: \\ $10.4 \%$ versus $9.3 \%, P=0.446$; vitamin $\mathrm{D}: 9.1 \%$ versus $9.1 \%, P=0.231$ ). \\ Conclusion: This study revealed that supplemental calcium and vitamin D intake among nephrolithiasis patients had no significant \\ associations with history of kidney stones. Those patients, especially those taking calcium supplements should be counseled to \\ monitor regularly their calcium levels to avoid hypercalciuria and consequent stone formation. \\ Keywords: Calcium, vitamin D, Supplements, Nephrolithiasis, Kidney, Stones \\ Please cite this paper as: Akel MG, Fahs IM. Calcium and vitamin D supplements administration in patients with nephrolithiasis; \\ an observational prospective study. J Parathyr Dis. 2018;6(2):39-41. DOI: 10.15171/jpd.2018.15. \\ Copyright (c) 2018 The Author(s); Published by Nickan Research Institute. This is an open-access article distributed under the terms \\ of the Creative Commons Attribution License, which permits unrestricted use, distribution, and reproduction in any medium, \\ provided the original work is properly cited.
}

\section{Introduction}

Vitamin supplements have become increasingly popular around the globe. Almost 50\% of individuals (50 years of age or older) regularly consume vitamin supplements in United States, among which calcium and vitamin D are the most frequent (1). However, large-scale randomized trials have revealed that vitamin supplements may be deleterious to health. For instance, calcium and vitamin D supplements have been linked to nephrolithiasis (2-4). Nephrolithiasis (kidney stones) is a common clinical disorder whose prevalence in the United States has doubled over the past three decades. This increase has also been noted in most European countries and Southeast Asia (5). More than $80 \%$ of kidney stones include calcium with the most being composed primarily of calcium oxalate (6). One of the main risk factors for calcium stone development is hypercalciuria (7) which might be elevated by higher levels of vitamin $\mathrm{D}$, as well (8). However, most of the observational studies do not support a significant association between higher nutritional vitamin $\mathrm{D}$ store and increased risk of stone formation. In addition, the likelihood that calcium supplements use increases stone formation is still controversial (9).

\section{Objectives \\ In the light of such contradictory data, we conducted the following study among Lebanese individuals to analyze the utilization of these supplements and to assess the presence of significant association with patients having nephrolithiasis.}

\section{Patients and Methods \\ Study population}

This is a descriptive observational study carried out at nephrology clinics selected randomly from the six Lebanese districts between March and July 2017.

\section{Ethical issues}

The research followed the tenets of the Declaration of Helsinki. The study was approved by the ethical committee 
Implication for health policy/practice/research/ medical education

Data regarding calcium and vitamin D supplements among patients with nephrolithiasis are in consistent. Those patients should be counseled to monitor regularly their calcium levels to avoid hypercalciuria and consequent stone formation.

at the Lebanese International University. Written informed consents were obtained from all participants prior to enrollment.

\section{Statistical analysis}

Data analysis was conducted with the statistical software package "Statistical Package for Social Sciences" software (SPSS, version 22). Descriptive statistics were used to describe patient characteristics and mean for continuous variables. The studied groups were compared for statistically significant associations using chi-square test. All reported $P$ values were two-sided with the alpha set at a significance of 0.05 .

Results

Around 550 patients were randomly approached and interviewed. Table 1 describes the participants' baseline characteristics. The mean age of the studied population was $42.12 \pm 15.59$ years with $51.5 \%$ (283) males and $48.5 \%$ (267) females. Almost half of the participants had a history of kidney stones $(53.8 \%, 296)$. Calcium and vitamin D supplements were utilized by $19.6 \%$ (108) and $18.2 \%$ (100) (Figure 1), respectively. Calcium and vitamin D supplements intake were not associated significantly with history of kidney stones (calcium: 10.4\% [57] had kidney stones versus 9.3\% [51] did not have kidney stones, $P=0.446$; vitamin D: 9.1\% [50] had kidney stones vs. 9.1\% [50] did not have kidney stones, $P=0.231$ ).

Table 1. Baseline characteristics of the studied participants

\begin{tabular}{lc}
\hline Variable & \\
\hline Age, mean \pm SD & $42.12 \pm 15.59$ \\
Gender, No. (\%) & $283(51.5)$ \\
$\quad$ Male & $267(48.5)$ \\
$\quad$ Female & \\
Socio-economic status, No. (\%) & $72(13.1)$ \\
Low income & $310(56.4)$ \\
Moderate income & $168(30.5)$ \\
High income & $19(3.5)$ \\
Education, No. (\%) & $145(26.4)$ \\
Illiterate & $163(29.6)$ \\
Elementary & $223(40.5)$ \\
High school & $296(53.8)$ \\
University & \\
Personal history of kidney stones, No. (\%) &
\end{tabular}

Discussion

Studies on calcium and vitamin D supplements intake have emerged conflicting results concerning their association with nephrolithiasis.

One-fifth of the participants in this study reported using calcium supplements with no significant association with those having a history of kidney stones. It is wellknown that calcium absorption through the intestine is important in renal stones development (10). The proportions of calcium absorption from dietary and supplemental sources tend to be higher among those with nephrolithiasis history (10).

However, dietary calcium restriction is no longer recommended in patients with kidney stones. Higher incidence of stone formation was associated with lower calcium intake, according to a huge epidemiological study carried-out among healthy men (11). Similar results were obtained in the nurses health studies I and II $(3,12)$ and in the women's health initiative (10). Such unexpected effect is due to increased levels in urinary oxalate secondary to its reduced binding to calcium in the gastrointestinal tract. On the contrary, the results of studies on supplemental calcium intake is still controversial. The risk of stone development was elevated by $17 \%$ among women randomized to calcium and vitamin D supplements versus placebo in women's health initiative study (2) and by $20 \%$ in the nurse's health study I (3). On the other hand, other studies such as nurse's health study II and the health professionals follow up study revealed the absence of an association between calcium supplements intake and kidney stones development (12-14). Hence, patients with nephrolithiasis should be counseled to increase their dietary calcium resources and to routinely monitor their urine if they are using calcium supplements. If hypercalcemia occurred, evaluation of the time of administration, dose and type of calcium supplements should be undertaken.

Almost one-fifth, also, of the participants revealed taking vitamin D supplements with no significant association with patients having nephrolithiasis. Vitamin D supplements use in kidney stones-diseased patients do not

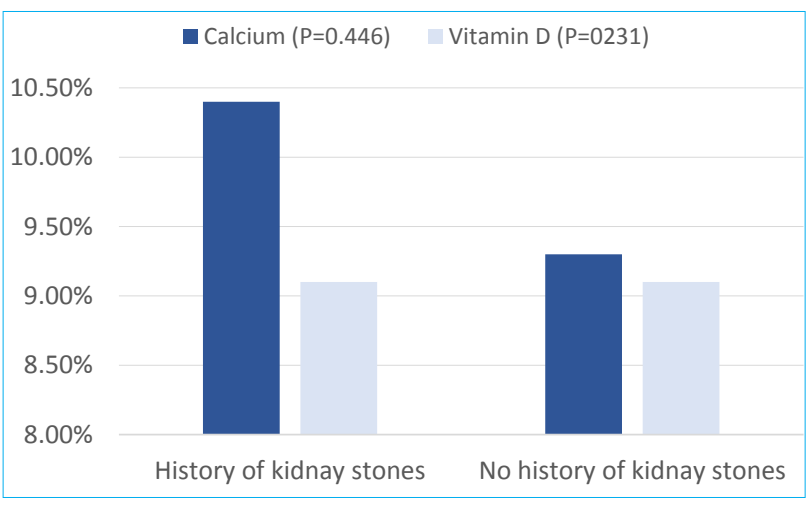

Figure 1. Calcium and vitamin D supplements intake among participants with and without history of kidney stones. 
provide consistent recommendations. The risk of kidney stones formation was $73 \%$ higher among those with circulating calcitriol levels in the highest quartile (4). In contrast, another study revealed no significant association between nephrolithiasis and total and supplemental vitamin D intake (15). Similarly, average urinary calcium excretion was not elevated significantly with vitamin D3 supplements intake among the 138 individuals included in the study conducted by Aloia et al (16). However, two recent studies have found an inverse relationship between vitamin $\mathrm{D}$ and kidney stones. The lower the vitamin D level, the higher the risk for kidney stones $(17,18)$. According to the study of Ticinesi and colleagues (18), those with vitamin $\mathrm{D}$ deficiency were more than twice as likely to develop a kidney stone.

\section{Conclusion}

Despite no clear and consistent data regarding calcium and vitamin D supplements use among kidney stones-diseased patients, recent studies suggested no increased incidence of kidney stones with calcium supplements along with a protective dietary calcium role. As for vitamin $\mathrm{D}$, a new inverse relationship was established.

This study revealed that supplemental calcium and vitamin $\mathrm{D}$ intake among nephrolithiasis patients have no significant associations with history of kidney stones. Hence, these patients, especially those taking calcium supplements should be counseled to monitor regularly their calcium levels to avoid hypercalciuria and consequent stone formation.

\section{Acknowledgments}

We thank the patients for agreeing to participate in the study.

\section{Authors' contributions}

MA and IF contributed equally toward study design, data collection, analysis and interpretation, manuscript drafting and write-up of the paper. Both authors read and approved the final manuscript.

\section{Conflicts of interest}

The authors declare that they have no conflict of interests.

\section{Ethical considerations}

Ethical issues (including plagiarism, data fabrication, double publication) have been completely observed by the authors.

\section{Funding/Support}

This research received no specific grant from any funding agency in the public, commercial, or not-for-profit sectors.

\section{References}

1. Bailey RL, Gahche JJ, Lentino CV, Dweyer JT, Engel JS, Thomas PR, et al. Dietary supplement use in the United
States. 2003-2006. J Nutr. 2011;141:261-6.

2. Jackson RD, LaCroix AZ, Gass M, Wallace RB, Robbins J, Lewis CE, et al. Calcium plus vitamin D supplementation and the risk of fractures. N Engl J Med. 2006;354:669-83.

3. Curhan GC, Willett WC, Speizer FE, Spiegelman D, Stampfer MJ. Comparison of dietary calcium with supplemental calcium and other nutrients as factors affecting the risk for kidney stones in women. Ann Intern Med. 1997;126:497-504.

4. Taylor EN, Hoofnagle AN, Curhan GC. Calcium and phosphorus regulatory hormones and risk of incident symptomatic kidney stones. Clin J Am Soc Nephrol. 2015; 10:667-75.

5. Romero V, Akpinar H, Assimos DG. Kidney stones: a global picture of prevalence, incidence, and associated risk factors. Rev Urol. 2010;12:e86-96.

6. Saigal CS, Joyce G, Timilsina AR. Urologic diseases in America project. Kidney Int. 2005;68:1808-14.

7. Curhan GC, Taylor EN. 24-h uric acid excretion and the risk of kidney stones. Kidney Int. 2008;73:489-96.

8. Shakhssalim N, Gilani KR, Parvin M, Torbati PM, Kashi $\mathrm{AH}$, Azadvari $\mathrm{M}$, et al. An assessment of parathyroid hormone, calcitonin, 1,25 $(\mathrm{OH}) 2$ vitamin $\mathrm{D} 3$, estradiol and testosterone in men with active calcium stone disease and evaluation of its biochemical risk factors. Urol Res. 2011;39:1-7.

9. Sorensen MD. Calcium intake and urinary stone disease. Transl Androl Urol. 2014;3:235-240.

10. Sorensen MD, Kahn AJ, Reiner AP, Tsenq TY, Shikany JM, Wallace RB, et al. Impact of nutritional factors on incident kidney stone formation: a report from the WHI OS. J Urol 2012; 187:1645-9.

11. Curhan GC, Willet WC, Rimm EB, Stampfer MJ. A prospective study of dietary calcium and other nutrients and the risk of symptomatic kidney stones. N Engl J Med. 1983;328:833-8.

12. Curhan GC, Willett WC, Knight EL, Stampfer MJ. Dietary factors and the risk of incident kidney stones in younger women: Nurses' Health Study II. Arch Intern Med. 2004;164:885-91.

13. Curhan GC, Willett WC, Rimm EB, Stampfer MJ. A prospective study of dietary calcium and other nutrients and the risk of symptomatic kidney stones. N Engl J Med. 1993; 328:833-8.

14. Taylor EN, Stampfer MJ, Curhan GC. Dietary factors and the risk of incident kidney stones in men: new insights after 14 years of follow-up. J Am Soc Nephrol 2004;15:3225-32.

15. Ferraro P, Taylor E, Gambaro G, et al. Vitamin D Intake and the Risk of Incident Kidney Stones. Journal Urol. 2017;197:405-10.

16. Aloia JF, Patel M, Dimaano R, Li-Nq M, Talwar SA, Mikhail $M$, et al. Vitamin $D$ intake to attain a desired serum 25-hydroxyvitamin D concentration. Am J Clin Nutr. 2008;87:1952-8.

17. Girón-Prieto $M$, del Carmen Cano-García M, ArrabalPolo M, Poyatos-Andujar, Quesada-Charneco M, de HaroMunoz T, et al. Analysis of vitamin D deficiency in calcium stone-forming patients. Int Urol Nephrol. 2016;48:12431246.

18. Ticinesi A, Nouvenne A, Ferraro P, Folesani G, Lauretani F, Allegri F, et al. Idiopathic Calcium Nephrolithiasis and Hypovitaminosis D: A Case-control Study. Urology. 2016;87:40-45. 